\title{
Transport through Andreev bound states in a graphene quantum dot
}

\author{
Travis Dirks, Taylor L. Hughes, Siddhartha Lal ${ }^{\dagger}$, Bruno Uchoa, Yung-Fu Chen, Cesar Chialvo, \\ Paul M. Goldbart ${ }^{\dagger}$ and Nadya Mason*
}

\begin{abstract}
When a low-energy electron is incident on an interface between a metal and superconductor, it causes the injection of a Cooper pair into the superconductor and the generation of a hole that reflects back into the metal-a process known as Andreev reflection. In confined geometries, this process can give rise to discrete Andreev bound states (ABS), which can enable transport of supercurrents through nonsuperconducting materials and have recently been proposed as a means of realizing solid-state qubits ${ }^{1-3}$. Here, we report transport measurements of sharp, gate-tunable ABS formed in a superconductor-quantum dot (QD)-normal system realized on an exfoliated graphene sheet. The QD is formed in graphene beneath a superconducting contact as a result of a workfunction mismatch ${ }^{4,5}$. Individual ABS form when the discrete QD levels are proximity-coupled to the superconducting contact. Owing to the low density of states of graphene and the sensitivity of the QD levels to an applied gate voltage, the ABS spectra are narrow and can be continuously tuned down to zero energy by the gate voltage.
\end{abstract}

Although signatures of Andreev reflection and bound states in conductance have been widely reported ${ }^{6}$, it has been difficult to directly probe individual ABS. In superconductor (SC)-graphene structures, most previous work has focused on the nature of the supercurrent in well-coupled Josephson junctions ${ }^{7-10}$. SC-QD hybrids in graphene have not been studied, although recent work has predicted ${ }^{11-14}$ and demonstrated ${ }^{15}$ that ABS can be isolated by coupling them to discrete QD energy levels. However, the ABS peaks in previous SC-QD experiments were strongly broadened, either by the large lead density of states ${ }^{15}$ or by the lack of a tunnel barrier ${ }^{16}$. In the work described in this Letter, sharp subgap conductance peaks are obtained by tunnelling into a proximity-coupled QD formed within graphene, a high-mobility zero-gap semiconductor ${ }^{17}$. We focus on the two lowest-energy conductance peaks that occur below the superconducting gap, and show that they are a signature of transport by means of ABS. The spectral pattern of these peaks as a function of gate and bias voltage is consistent with a simple theoretical model of ABS spectra presented below, and can be accurately fitted with a more detailed microscopic calculation.

The data shown in this Letter were taken from one single-layer graphene device (sample A) and one multilayer device (sample B, approximately ten layers thick). Similar behaviour was seen in three other devices (two single layer and one bilayer). As the features are robust on adding layers, it is evident that a precise Dirac-point band structure is not a requirement. The sample geometry and measurement circuit are shown in Fig. 1a; the location of the quantum dot that forms beneath the SC probe is depicted in
Fig. 2a (discussed below). The devices consist of normal-metal end contacts $(2 \mathrm{~nm} / 50 \mathrm{~nm} \mathrm{Cr} / \mathrm{Au}$ bilayer $)$ and SC tunnel probes ${ }^{18,19}$ $(200 \mathrm{~nm} / 30 \mathrm{~nm} \mathrm{~Pb} / \mathrm{In}$ bilayer) (see Methods for details). The tunnelling resistances through the SC probes were typically 10-100 times larger than the end-to-end resistances.

In all samples, the charge neutral point, as seen in the end-to-end conductance versus back-gate measurements, shows a large offset to the positive-gate side (see Fig. 1c,d for samples A and B, respectively). For example, sample A shows an asymmetric cone around the Dirac point at $V_{\mathrm{g}} \sim+17.5 \mathrm{~V}$. The asymmetry and direction of offset are consistent with a work-function mismatch $(\Delta W)$ at the $\mathrm{Cr} / \mathrm{Au}$-graphene interface, which results in a transfer of charge that equalizes the surface potentials ${ }^{4}$; similar charge transfer effects have previously been predicted ${ }^{4}$ and observed ${ }^{5}$. Because of its low density of states, graphene is efficiently doped by this charge transfer. The $\mathrm{Cr} / \mathrm{Au}$-graphene interface of the end contacts is dominated by the work function of the Au. (This is expected because the Cr layer is so thin as to be discontinuous. It is also similar to the $\mathrm{Ti} / \mathrm{Au}$-graphene contacts measured in ref. 5.) Therefore, $\Delta \mathrm{W}$ ranges from 0.14 to $1.04 \mathrm{eV}$ (see Methods for calculation). The positive $\Delta \mathrm{W}$ indicates hole (that is, p-type) doping of the graphene under the end leads.

The superconducting tunnel probe also leads to a local doping of graphene. However, for the $\mathrm{Pb}$-graphene interface, $\Delta \mathrm{W}$ is -1.15 to $-0.25 \mathrm{eV}$ and is negative, implying local electron (n-type) doping. As illustrated in Figs $1 \mathrm{~b}$ and $2 \mathrm{a}$, the $\Delta \mathrm{W}$ doping generates a potential well underneath the SC tunnel probe, which acts as a confining potential for a QD - that is, a pn junction. Quantum dots formed by pn junctions have been observed in carbon nanotubes ${ }^{20}$, by means of work-function doping, and in graphene ${ }^{21}$, where Klein tunnelling through smooth barriers can lead to the confinement of carriers ${ }^{22,23}$. The QD is also proximity coupled to the superconducting lead. The interplay of the resulting Andreev reflections with Coulomb charging effects gives rise to low-energy ABS (see Fig. 2b). The ABS appear as subgap conductance peaks in our tunnelling conductance measurements. Independent experimental results demonstrating individual Andreev bound states within a carbon nanotube QD have recently been obtained ${ }^{24}$

In Fig. 3a we show typical tunnelling conductance measurements at a fixed gate voltage. The conductance is dominated by the characteristic BCS shape of the superconducting density of states of $\mathrm{Pb}$, with the expected gap $2 \Delta=2.6 \mathrm{meV}$. Small peaks occur inside the gap, where we might expect conductance to be suppressed exponentially with the tunnel barrier thickness ${ }^{25}$. No conductance is observed around zero bias, implying that the tunnel barrier is not leaky. The two lowest-energy subgap peaks are symmetric in bias voltage and have strong temperature dependence; the peaks 


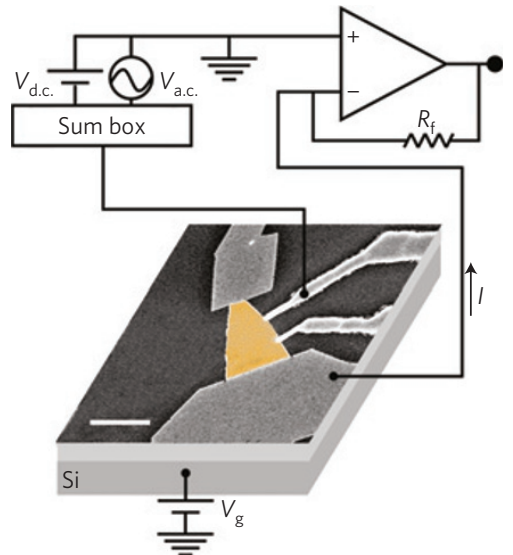

C

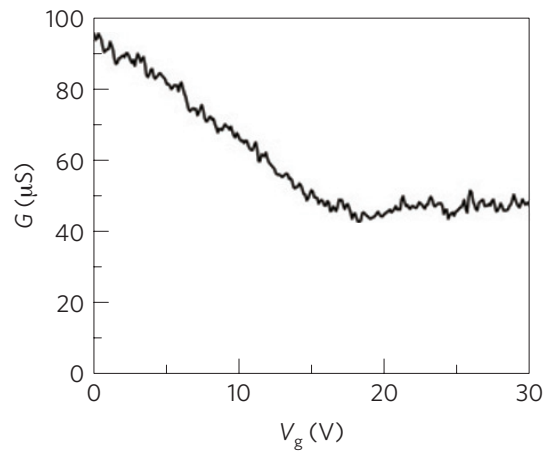

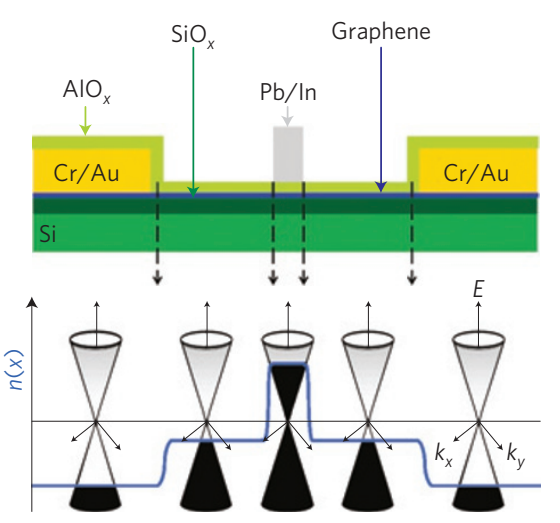

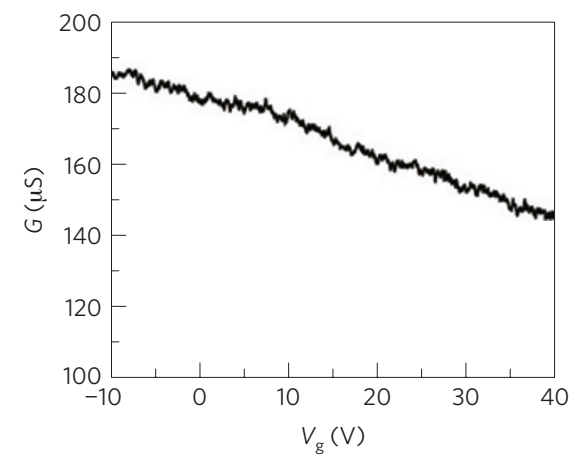

Figure 1 | Configuration and doping characteristics of the graphene device. a, Scanning electron micrograph of a device with an overlaid measurement circuit. Graphene is false coloured orange, large end contacts are $\mathrm{Cr} / \mathrm{Au}$ and middle tunnel probes are $\mathrm{Pb} / \mathrm{In}$. The scale bar is $5 \mu \mathrm{m}$. b, Above: side-view schematic diagram of the device. Below: Illustration of the doping profile as a function of position along the device (blue line) and Dirac cones showing the location of the Fermi level. The square well under the tunnel probe shows where $p-n$ junctions create a confining potential for the quantum dot.

c, End-to-end conductance versus back-gate for sample A (single layer), showing the Dirac cone. d, End-to-end conductance versus back-gate for sample B (multilayer). The asymmetry in $\mathbf{c}$ and $\mathbf{d}$ shows that the bulk graphene is $p$-doped by the back-gate.

decrease in amplitude as the temperature is raised to $\sim 0.8 \mathrm{~K}$, above which the amplitude remains constant. This behaviour is consistent with the temperature dependence of Coulomb-blockade peaks in a crossover from a quantum to a classical dot regime ${ }^{26}$. In addition to the lowest-energy subgap peaks, we observe an oscillatory contribution above and below the gap. The oscillations are due to geometric resonances between the end contacts and ABS states in the QD (discussed further in Supplementary Discussion, part I); if the QD could be connected directly to the Cr/Au leads, the lowest-energy subgap peaks would still appear, but not the oscillations ${ }^{24}$. The oscillations can be clearly distinguished from the lowest-energy subgap peaks by very different gate-voltage dependence, as discussed below.

Figure $4 \mathrm{a}$ shows a two-dimensional map of conductance versus bias and gate voltage for sample A. The lowest-energy subgap peaks show a striking gate- and bias-voltage-dependent pattern. Near zero gate voltage, the conduction peaks start to emerge from the SC gap edge. As the gate voltage becomes more negative, the peaks move towards zero bias and cross at $V_{\mathrm{g}} \approx-7 \mathrm{~V}$. As the gate voltage is further decreased, the peaks split and then begin to converge again below $V_{\mathrm{g}} \approx-10 \mathrm{~V}$. This pattern can be qualitatively explained as resonant transport through ABS levels (see Fig. 2b for a schematic diagram); the levels can be calculated from a simple phenomenological model (see below) and quantitatively fitted with detailed transport calculations (see Supplementary Discussion, part I), as shown in Fig. 4b. The correspondence between the calculation and the data for the lowest-energy subgap peaks is remarkable.

The appearance of subgap conductance peaks requires a competition between the charging energy $(U)$ and the effective superconducting pairing $\left(\Delta_{\text {eff }}\right)$ acting on the QD. We distinguish a
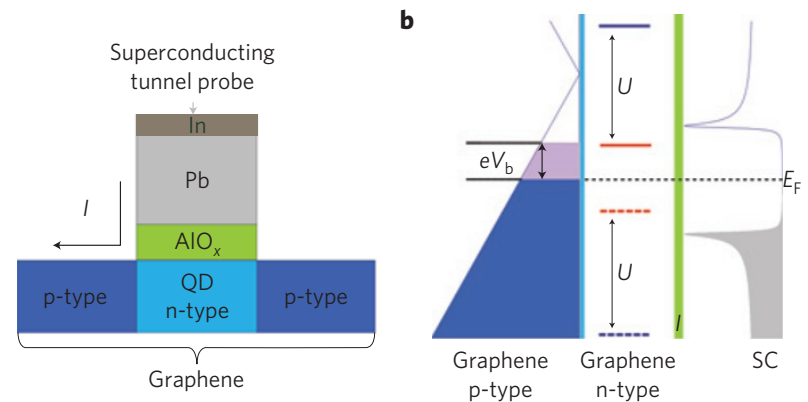

Figure 2 | Schematic diagrams of the graphene-QD-SC device.

a, Schematic diagram of the QD formed in graphene by a work-function mismatch at the $\mathrm{Pb}$ interface. $\mathbf{b}$, Schematic energy-level diagram of the graphene-QD-SC system. The density of states of the p-type graphene and SC tunnel probe is shown on the left and right, respectively, with filled states indicated. The $\mathrm{AlO}_{x}$ tunnel barrier is indicated in green on the right and the $\mathrm{p}-\mathrm{n}$ junction is indicated in light blue on the left. Blue/red energy levels refer to Andreev bound states. The solid (dashed) lines represent states that have dominant particle (hole) character. The bias voltage, $V_{b}$, is shown tuned to enable resonant subgap conduction.

between two physical regimes: (1) $U \ll \Delta_{\text {eff }}$ and (2) $U \gg \Delta_{\text {eff. }}$. In regime (1), the spin-up and spin-down states of the QD are nearly degenerate. As these levels are gated to within $\Delta_{\text {eff }}$ of the Fermi energy of the SC, they are occupied by paired electrons/holes, and the QD effectively becomes incorporated as part of the SC interface $^{26}$. The conductance is then Blonder-Tinkham-Klapwijklike and thus suppressed inside the gap, as in SC-normal interfaces 


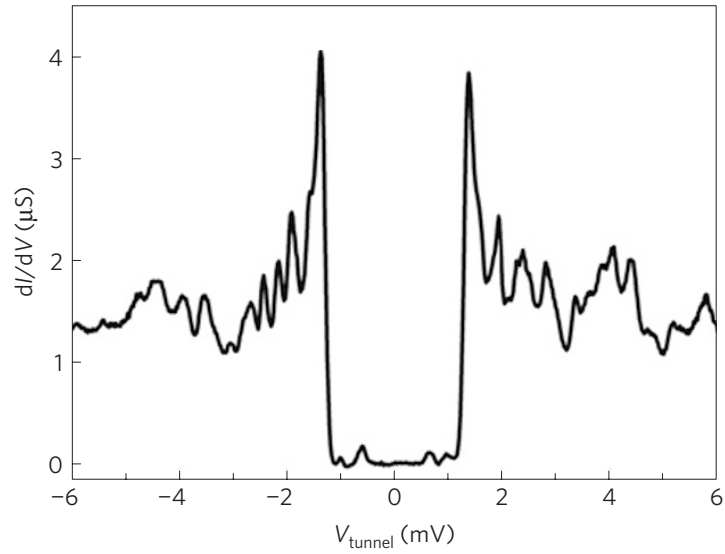

b

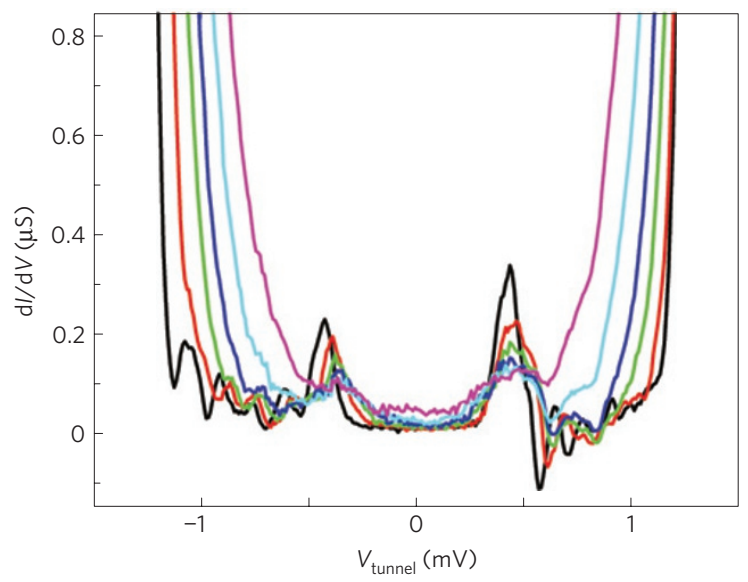

Figure 3 | Superconducting tunnelling data showing oscillations and subgap ABS peaks. a, Tunnelling differential conductance versus bias voltage (set-up as in Fig. 1a), for the multilayer graphene (sample B). Large conductance oscillations outside the gap are probably Fabry-Perot-like interference effects. Similar oscillations and subgap peaks are seen in sample A. Two ABS peaks are visible inside the SC gap. b, Temperature dependence of the subgap peaks. The temperature from the widest SC gap is $0.26,0.45,0.67,0.86,1.25$ and $1.54 \mathrm{~K}$. The peaks decrease in amplitude and increase in breadth as temperature is increased to $\sim 0.8 \mathrm{~K}$, then remain constant; this is consistent with a crossover from a quantum to a classical dot regime.

with large tunnel barriers ${ }^{25}$. In contrast, for regime (2), the charging energy dominates, and the spin-up and down states are widely split in energy, promoting pair-breaking. The QD then acts like a normal metal, and ABS are formed from the discrete QD states, owing to Andreev reflections at the SC-QD interface. Resonant transport through the ABS levels leads to the observed subgap conductance peaks (see Fig. 2b). The clear observation of the subgap features in the data suggests that our measurements are taken in regime (2). Further evidence for the existence of ABS in our system is provided by means of a calculation based on a microscopic Hamiltonian that describes a graphene quantum well that is proximity coupled to a superconducting lead (see Supplementary Discussion, part II).

A phenomenological model that considers the effect of the SC proximity coupling on a single pair of spin-split QD states explains the lowest-energy ABS physics. The effective Hamiltonian for a proximity-coupled QD is

$$
\begin{aligned}
H= & \left(\varepsilon_{\uparrow}-E_{\text {shift }}\right) \mathcal{c}_{\uparrow}^{\dagger} \mathcal{c}_{\uparrow}+\left(\varepsilon_{\uparrow}+U-E_{\text {shift }}\right) \mathcal{c}_{\downarrow}{ }^{\dagger} c_{\downarrow}+\Delta_{\text {eff }} \mathcal{c}_{\downarrow}^{\dagger} \mathcal{c}_{\uparrow}^{\dagger} \\
& +\Delta_{\text {eff }}{ }^{*} c_{\uparrow} c_{\downarrow}
\end{aligned}
$$
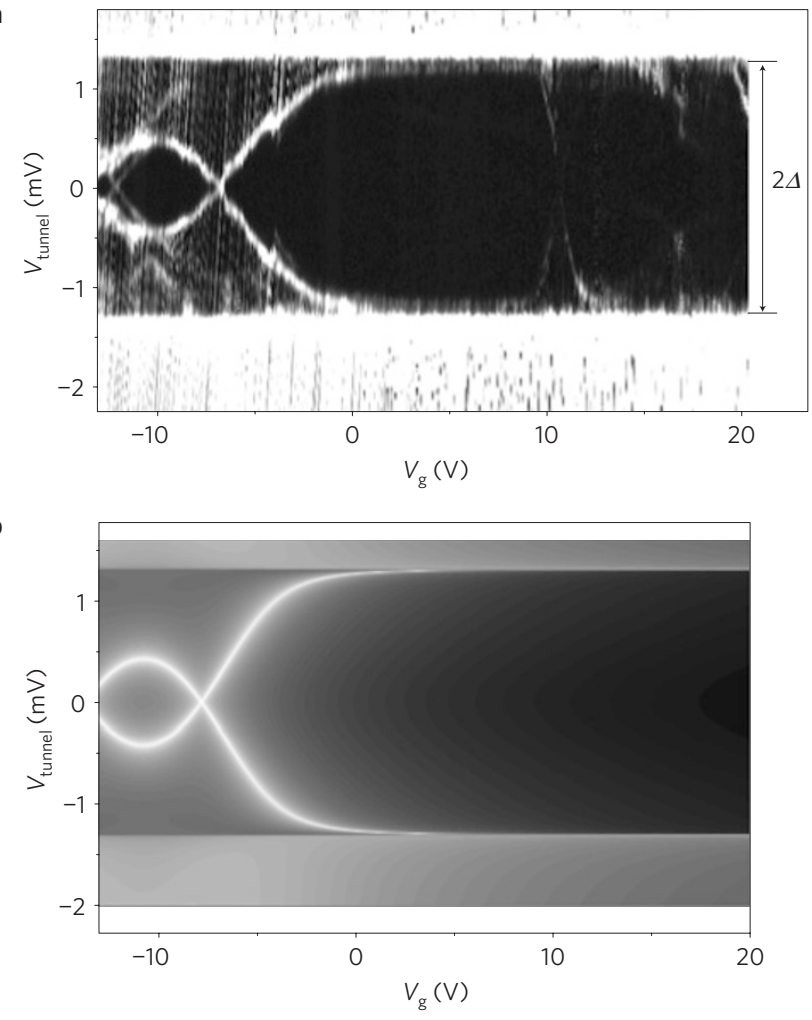

Figure 4 | Back-gate dependence of Andreev bound states.

a, Two-dimensional map of tunnelling differential conductance versus back-gate voltage ( $x$ axis) and bias voltage ( $y$ axis) on a log scale for the single-layer device (sample A). Bright white lines inside the gap (marked as $2 \Delta$ ) are subgap peaks, or ABS, which are symmetric about zero bias and gate dependent. b, A fit of the conductance data from the detailed transport calculations for a quantum dot with two levels, a finite charging energy and couplings to normal-metal and superconducting leads (see main text and Supplementary Discussion, part I).

where $E_{\text {shift }}$ represents the shift of the QD energy levels by the gate voltage, $\varepsilon_{\uparrow}, \varepsilon_{\uparrow}+U$ are the energies of the spin-split levels (we could equivalently choose $\left.\varepsilon_{\downarrow}, \varepsilon_{\downarrow}+U\right)$ and $c_{\sigma}\left(c_{\sigma}^{\dagger}\right)$ are creation (annihilation) fermionic operators having spin $\sigma=\uparrow, \downarrow$. For a QD of diameter $R \sim 100 \mathrm{~nm}$ (that is, roughly the size of the SC tunnel probe), the charging energy $U \sim e^{2} / \kappa R \sim 5 \mathrm{meV}$ (where $e$ is the electron charge and $\kappa=(1+\epsilon) / 2$, and the dielectric constant of $\left.\mathrm{SiO}_{2} \epsilon \approx 4\right)$. The resulting $\mathrm{ABS}$ energy levels lie at $E_{ \pm}=1 / 2\left( \pm U+\sqrt{4 \Delta_{\text {eff }}^{2}+\left(2 \varepsilon_{\uparrow}-2 E_{\text {shift }}+U\right)^{2}}\right)$. For $U=0$, the energies $E_{ \pm}$are larger than $\left|\Delta_{\text {eff }}\right|$ for all gate voltages, implying that the appearance of zero-bias subgap conductance features requires a non-zero charging energy. With increasing charging energy, a pair of zero-energy ABS appears at a critical value of $U, U_{\mathrm{c}}=2\left|\Delta_{\text {eff }}\right|$, on tuning the gate voltage such that $E_{\text {shift }}\left(V_{\mathrm{g}}\right)=\varepsilon_{\uparrow}+\Delta_{\text {eff. At }}$. At this gate voltage and $U_{c}$, the bound states are equal-amplitude superpositions of particle and hole states, and thus effectively charge neutral. At zero temperature, a quantum critical point separates the superconducting $\left(U<U_{\mathrm{c}}\right)$ and Coulomb-charging $\left(U>U_{\mathrm{c}}\right)$ regimes of the QD. This suggests the possibility of tuning through the quantum phase transition by changing the size of the QD.

For $U>U_{c}$, ABS appear within the gap, with the gate-voltage dependence illustrated in Fig. 5. Comparison with Fig. 4a shows that we observe the low-energy bound states and that $E_{-}$can be extracted from the data. For example, at $V_{\mathrm{g}}=-10 \mathrm{~V}, E_{-} \sim 0.5 \mathrm{meV}$. $U$ can also be extracted if both zero-bias crossing points are observed, though the accuracy is only as good as the knowledge of the gate capacitance (see Supplementary Discussion, part I). 

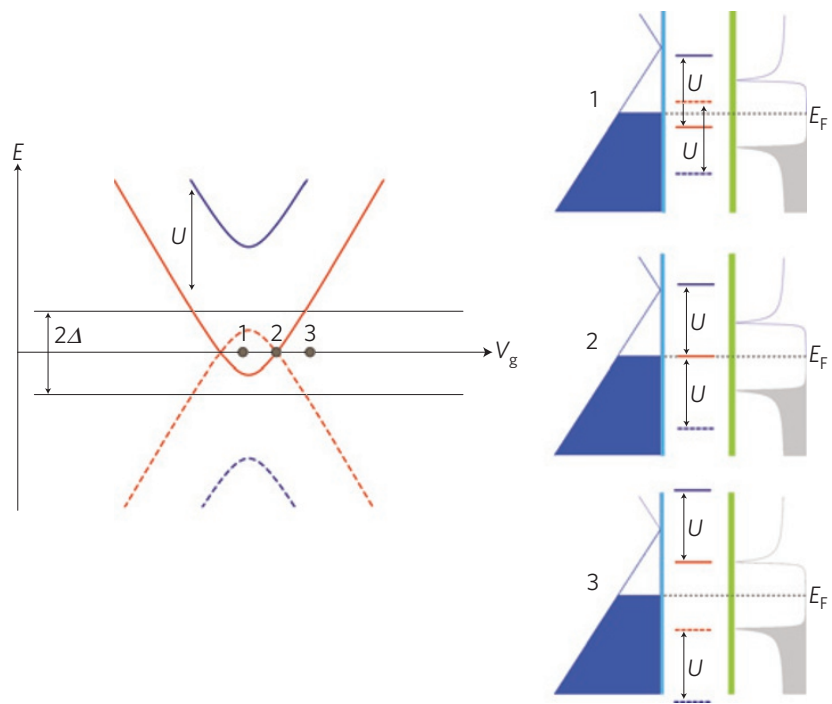

Figure 5 | Diagrams showing the energy dependence of Andreev bound states. Left: Energy diagram showing the evolution of $A B S$ levels in a quantum dot with varying gate voltage. $U$ is the charging energy of the quantum dot and $\Delta$ is the superconducting gap of the tunnel probe. Right: Three resonant tunnelling diagrams corresponding to the three different gate voltages marked on the left-hand diagram. The solid (dashed) lines represent states which have dominant particle (hole) character. The bias voltages required for conductance through the $A B S$ are the energy differences between the bound-state levels and $E_{\mathrm{F}}$ of the $\mathrm{SC}$. For point 1 , one Andreev bound state (red) is below the SC gap edge and one (blue) is above; this gives two subgap peaks (red levels) in the conductance at finite (positive and negative) bias voltage. At point 2, an ABS is tuned to zero energy, which leads to a zero-bias conductance peak. At point 3, the ABS level is at the gap edge, which leads to subgap peaks that are pushed towards the gap edge.

From the fit in Fig. 4b, we extract $U \sim 7.7 \mathrm{meV}$. Interestingly, Fig. 5 also shows that there are two gate voltages at which zero-energy bound states exist; the second crossing point lies just outside the gate-voltage range of our measurements. We note that, for the transport calculations, sharp ABS resonances could only be found when the normal (superconducting) lead coupled to the QD had relatively low (high) density of states; this implies that a low-density 'lead' such as graphene is indispensable for obtaining narrow ABS spectra. Such a sharp resolution of the levels-which have decoherence times inversely proportional to their width-may open a route towards applications, such as the design of quantum computing qubits in graphene-SC heterojunctions ${ }^{2,3}$.

\section{Methods}

The graphene samples were mechanically exfoliated onto highly doped Si substrates capped with $300 \mathrm{~nm} \mathrm{SiO}$. The graphene thickness was determined by means of optical microscopy, Raman spectroscopy and atomic force microscopy. The devices consist of four electrodes on a piece of graphene (see Fig. 1a). The two large end leads are $\mathrm{Cr} / \mathrm{Au}$ and the narrower middle probes are $\mathrm{Pb} / \mathrm{In}$. The end electrodes were patterned by conventional electron-beam lithography and electron-beam evaporation of $2 \mathrm{~nm} \mathrm{Cr}$ followed by $50 \mathrm{~nm} \mathrm{Au}$. The chips were then annealed in $\mathrm{H}_{2}$ and $\mathrm{Ar}$ at $300^{\circ} \mathrm{C}$ for $2 \mathrm{~h}$. Next, the devices were covered in 12 layers of $\mathrm{AlO}_{x}$ by means of atomic-layer deposition, and finally 200 -nm-wide SC tunnel probes were patterned by conventional electron-beam lithography and thermal evaporation of $200 \mathrm{~nm} \mathrm{~Pb}$ followed by $30 \mathrm{~nm} \mathrm{In}$. The In is used as a protective capping layer, and does not seem to affect the superconducting gap in the $\mathrm{Pb}$. The tunnelling resistances through the SC probes, $R_{\text {tunnel }} \sim 200-500 \mathrm{k} \Omega$, are typically $10-100$ times larger than the graphene's end-to-end resistances, $R_{\text {end-to-end }} \sim 5-20 \mathrm{k} \Omega$. Sample $A$ is a single-layer graphene device with a distance between the two end contacts $L \sim 4.2 \mu \mathrm{m}$, a width $W \sim 1.5 \mu \mathrm{m}$ and an SC probe junction size of $\sim 0.2 \times 0.2 \mu \mathrm{m}(0.3 \times 0.2 \mu \mathrm{m})$. Sample B is a multilayer device with $L \sim 6.4 \mu \mathrm{m}$, $W \sim 0.8 \mu \mathrm{m}$ and an SC probe junction $\sim 0.3 \times 0.2 \mu \mathrm{m}$. Measurements were made in a helium-3 cryostat using standard a.c. lock-in techniques. The measurement set-up is shown in Fig. la. Tunnelling differential conductance measurements were made by applying a sum of d.c. bias voltage $V_{\text {d.c. }}$ and a.c. excitation voltage $V_{\text {a.c. }}$ to the superconducting tunnel probe, and a voltage $V_{\mathrm{g}}$ to the back gate, while measuring the differential conductance $\mathrm{d} I_{\text {a.c. }} / \mathrm{d} V_{\text {a.c. }}$ at one of the graphene end contacts as illustrated in Fig. 1a.

The sign of the charge transfer due to work-function-mismatch doping is the same as that of the mismatch $\Delta W=W_{\mathrm{m}}-W_{\mathrm{g}}-W_{\mathrm{c}}$, where $W_{\mathrm{m}}$ is the work function of the metal, $W_{\mathrm{g}}=4.5 \mathrm{eV}$ is the work function of the graphene ${ }^{4}$ and $W_{\mathrm{c}} \sim 0-0.9 \mathrm{eV}$ is an effective potential that arises from metal-graphene chemical interactions ${ }^{4}$ For the $\mathrm{Cr} / \mathrm{Au}$-graphene interface of the end contacts (where the work function of the thick Au layer dominates that of the thin $\mathrm{Cr}$ ), $W_{\mathrm{Au}}=5.54 \mathrm{eV}$ so $\Delta W=0.14-1.04 \mathrm{eV}$. For the SC tunnel probe-graphene interface, $W_{\mathrm{Pb}}=4.25 \mathrm{eV}$ so $\Delta W=-1.15$ to $-0.25 \mathrm{eV}$. For the work function of the metals, we use standard theoretical values (see CRC handbook, 2008), which are consistent with known experimental results.

Received 12 May 2010; accepted 21 December 2010; published online 6 February 2011

\section{References}

1. Shafranjuk, S. E., Nevirkovets, I. P. \& Ketterson, J. B. A qubit device based on manipulations of Andreev bound states in double-barrier Josephson junctions. Solid State Commun. 121, 457-460 (2002).

2. Zazunov, A., Shumeiko, V. S., Bratus', E. N., Lantz, J. \& Wendin, G. Andreev level qubit. Phys. Rev. Lett. 90, 087003 (2003).

3. Zazunov, A., Shumeiko, V. S., Wendin, G. \& Bratus', E. N. Dynamics and phonon-induced decoherence of Andreev level qubit. Phys. Rev. B 71, 214505 (2005).

4. Giovannetti, G. et al. Doping graphene with metal contacts. Phys. Rev. Lett. 101, 026803 (2008).

5. Huard, B., Stander, N., Sulpizio, J. A. \& Goldhaber-Gordon, D. Evidence of the role of contacts on the observed electron-hole asymmetry in graphene. Phys. Rev. B 78, 121402 (2008).

6. Deutscher, G. Andreev-Saint-James reflections: A probe of cuprate superconductors. Rev. Mod. Phys. 77, 109-135 (2005).

7. Heersche, H. B., Jarillo-Herrero, P., Oostinga, J. B., Vandersypen, L. M. K. \& Morpurgo, A. F. Induced superconductivity in graphene. Solid State Commun 143, 72-76 (2007)

8. Ojeda-Aristizabal, C., Ferrier, M., Gueron, S. \& Bouchiat, H. Tuning the proximity effect in a superconductor-graphene-superconductor junction. Phys. Rev. B 79, 165436 (2009).

9. Du, X., Skachko, I. \& Andrei, E. Y. Josephson current and multiple Andreev reflections in graphene SNS junctions. Phys. Rev. B 77, 184507 (2008).

10. Beenakker, C. W. J. Specular Andreev reflection in graphene. Phys. Rev. Lett. 97, 067007 (2006)

11. Meng, T., Florens, S. \& Simon, P. Self-consistent description of Andreev bound states in Josephson quantum dot devices. Phys. Rev. B 79, 224521 (2009).

12. Sköldberg, J., Löfwander, T., Shumeiko, V.S. \& Fogelström, M. Spectrum of Andreev bound states in a molecule embedded inside a microwave-excited superconducting junction. Phys. Rev. Lett. 101, 087002 (2008).

13. Zhang, Z.Y. Differential conductance through a NINS junction on graphene. J. Phys. Condens. Matter 20, 445220 (2008).

14. Ossipov, A., Titov, M. \& Beenakker, C. W. J. Reentrance effect in a graphene $\mathrm{n}-\mathrm{p}-\mathrm{n}$ junction coupled to a superconductor. Phys. Rev. B 75, 241401 (2007).

15. Deacon, R. S. et al. Tunneling spectroscopy of Andreev energy levels in a quantum dot coupled to a superconductor. Phys. Rev. Lett. 104, 076805.

16. Eichler, A. et al. Even-odd effect in Andreev transport through a carbon nanotube quantum dot. Phys. Rev. Lett. 99, 126602 (2007).

17. Geim, A. K. \& Novoselov, K. S. The rise of graphene. Nature Mater. 6, 183-191 (2007).

18. Chen, Y. F., Dirks, T., Al-Zoubi, G., Birge, N. O. \& Mason, N. Nonequilibrium tunneling spectroscopy in carbon nanotubes. Phys. Rev. Lett. 102, 036804 (2009)

19. Dirks, T., Chen, Y. F., Birge, N. O. \& Mason, N. Superconducting tunnelling spectroscopy of a carbon nanotube quantum dot. Appl. Phys. Lett. 95, 192103 (2009).

20. Park, J. \& McEuen, P. L. Formation of a p-type quantum dot at the end of an n-type carbon nanotube. Appl. Phys. Lett. 79, 1363-1365 (2001).

21. Young, A. F. \& Kim, P. Quantum interference and Klein tunnelling in graphene heterojunctions. Nature Phys. 5, 222-226 (2009).

22. Shytov, A. V., Rudner, M. S. \& Levitov, L. S. Klein backscattering and Fabry-Perot interference in graphene heterojunctions. Phys. Rev. Lett. 101, 156804 (2008).

23. Silvestrov, P. G. \& Efetov, K. B. Quantum dots in graphene. Phys. Rev. Lett. 98, 016802 (2007).

24. Pillet, J-D. et al. Andreev bound states in a supercurrent-carrying carbon nanotube revealed. Nature Phys. 6, 965-969 (2010). 
25. Blonder, G. E., Tinkham, M. \& Klapwijk, T. M. Transition from metallic to tunnelling regimes in superconducting microconstrictions: Excess current, charge imbalance, and supercurrent conversion. Phys. Rev. B 25, 4515-4532 (1982).

26. Kouwenhoven, L. P. et al. in NATO Advanced Study Institute on Mesoscopic Electron Transport E345 edn (eds Sohn, L. L., Kouwenhoven, L. P. \& Schön, G.) 105-214 (1997).

\section{Acknowledgements}

This research was supported by the Department of Energy Division of Materials Science under grant DE-FG02-07ER46453 through the Frederick Seitz Materials Research Laboratory at the University of Illinois at Urbana-Champaign, and partly carried out in the Materials Research Laboratory Central Facilities (partially supported by the Department of Energy under DE-FG02-07ER46453 and DE-FG02-07ER46471). T.L.H. acknowledges the National Science Foundation under grant DMR-0758462 and the
Institute for Condensed Matter Theory at University of Illinois at Urbana-Champaign, and B.U. acknowledges the Department of Energy under grant DE-FG02-91ER45439. We acknowledge conversations with J. Maciejko.

\section{Author contributions}

T.D. and Y-F.C. carried out the experiments. C.C. helped fabricate the samples. T.D., T.L.H., S.L., B.U., P.M.G. and N.M. analysed the data and wrote the main paper. T.L.H., B.U. and P.M.G. wrote Supplementary Information.

\section{Additional information}

The authors declare no competing financial interests. Supplementary information accompanies this paper on www.nature.com/naturephysics. Reprints and permissions information is available online at http://npg.nature.com/reprintsandpermissions. Correspondence and requests for materials should be addressed to N.M. 